\title{
ARTICLE The role of dopamine dysregulation and evidence for the transdiagnostic nature of elevated dopamine synthesis in psychosis: a positron emission tomography (PET) study comparing schizophrenia, delusional disorder, and other psychotic disorders
}

Pak Wing Calvin Cheng (D) ${ }^{1}$, Wing Chung Chang ${ }^{1}$, Gladys G. Lo ${ }^{2}$, Kit Wa Sherry Chan ${ }^{1}$, Ho Ming Edwin Lee ${ }^{1}$, Lai Ming Christy Hui ${ }^{1}$, Yi Nam Suen (D)', Yim Lung Eric Leung ${ }^{2}$, Kai Ming Paul Au Yeung ${ }^{2}$, Sirong Chen ${ }^{2}$, Ka Fung Henry Mak ${ }^{3}$, Pak Chung Sham ${ }^{1}$, Barbara Santangelo ${ }^{4,5}$, Mattia Veronese $\mathbb{D}^{5}$, Chi-Lai Ho ${ }^{2}$, Yu Hai Eric Chen ${ }^{1,6}$ and Oliver D. Howes ${ }^{4,7,8}$

There have been few studies performed to examine the pathophysiological differences between different types of psychosis, such as between delusional disorder (DD) and schizophrenia (SZ). Notably, despite the different clinical characteristics of DD and schizophrenia (SZ), antipsychotics are deemed equally effective pharmaceutical treatments for both conditions. In this context, dopamine dysregulation may be transdiagnostic of the pathophysiology of psychotic disorders such as DD and SZ. In this study, an examination is made of the dopamine synthesis capacity (DSC) of patients with SZ, DD, other psychotic disorders, and the DSC of healthy subjects. Fifty-four subjects were recruited to the study, comprising 35 subjects with first-episode psychosis (11 DD, 12 SZ, 12 other psychotic disorders) and 19 healthy controls. All received an ${ }^{18} \mathrm{~F}$-DOPA positron emission tomography (PET)/magnetic

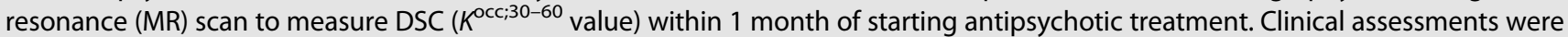
also made, which included Positive and Negative Syndrome Scale (PANSS) measurements. The mean $K^{\circ c c ; 30-60}$ was significantly greater in the caudate region of subjects in the DD group (ES $=0.83$, corrected $p=0.048$ ), the SZ group ( $E S=1.40$, corrected $p=$ 0.003 ) and the other psychotic disorder group ( $E S=1.34$, corrected $p=0.0045)$, compared to that of the control group. These data indicate that DD, SZ, and other psychotic disorders have similar dysregulated mechanisms of dopamine synthesis, which supports the utility of abnormal dopamine synthesis in transdiagnoses of these psychotic conditions.

Neuropsychopharmacology (2020) 45:1870-1876; https://doi.org/10.1038/s41386-020-0740-x

\section{INTRODUCTION}

The term "psychosis" encompasses a heterogeneous group of disorders that are all typified by patients demonstrating psychotic symptoms such as delusions and hallucinations. The pathophysiologies underlying these diverse disorders may share common mechanisms, but the relationship between these disorders, and particularly between delusional disorder (DD) and schizophrenia (SZ), is still under debate.

DD has long been recognized as a discrete diagnostic entity within a spectrum of psychotic disorders. It was first described by Kraepelin as "paranoia" [1]. The first operationalized diagnostic classifications to include this disorder were the Diagnostic and Statistical Manual (DSM)-IIIR and the International Statistical Classification of Diseases and Related Health Problems, 10th revision (ICD-10). Currently, DD is classified as an illness characterized by at least 1 month of delusions but no other psychotic symptoms, according to the American Psychiatric Association's Diagnostic and Statistical Manual of Mental Disorders, Fifth Edition (DSM-5) [2]. Patients who suffer from other nonaffective psychoses that cannot be classified as SZ or DD are diagnosed with unspecified SZ spectrum, brief psychotic disorder, or other psychotic disorder, in accordance with the DSM-5 criteria [2]. DD is considered a monosymptomatic condition because its key presentation involves a single, well-structured, complex delusional belief that is manifest without prominent hallucinations. Patients with this condition lack other features commonly observed in SZ, such as negative symptoms and formal thought disorders. "Non-bizarre" delusions were previously included among the criteria for DD, but this criterion has since been removed. DD is a relatively rare psychotic disorder, with lifetime DD prevalences of $0.18 \%$ [3] and $0.15 \%$ [4] found from population-based surveys. DD has been associated with cognitive impairments in memory, verbal learning,

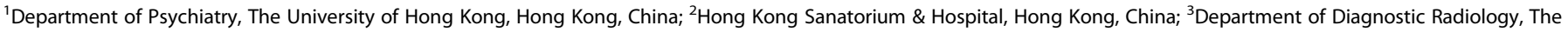

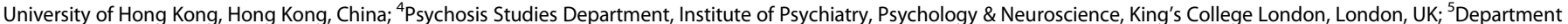

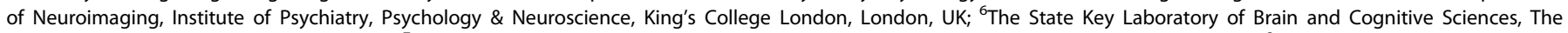

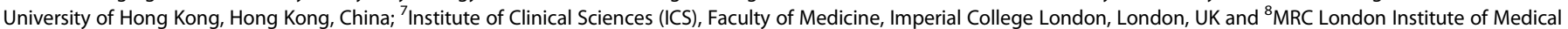
Sciences, Hammersmith Hospital, London, UK Correspondence: Yu Hai Eric Chen (eyhchen@hku.hk)
} 
executive function, and working memory and may therefore further reduce the quality of life of the patient [5].

The identity of DD as a valid and distinct psychiatric entity, or as a subtype of SZ, has been debated since its initial discovery by Kraepelin. DD has been distinguished from SZ since the publication of the DSM-IIIR, and the latest diagnostic criteria in the DSM-5 and ICD-11 have maintained this classification. This diagnostic distinction is supported by previous observations that the clinical features of DD and SZ are often differentiable in terms of sex, age of onset, and functionality [6-8]. It was also found in a family history study that the prevalence of SZ in the relatives of DD probands was significantly lower than in the relatives of SZ probands [9], reinforcing that DD is a distinct entity from SZ.

Despite the differences in the clinical characteristics of DD and $S Z$, antipsychotic drugs targeting the dopamine pathway have been deemed equally effective for the pharmaceutical treatment of both conditions [10-13]. Data from a 12-week prospective follow-up study showed that there were similar responses to antipsychotics in patients with DD and SZ, suggesting that these disorders share a common underlying pathophysiology involving dopamine dysfunction. SZ has been studied with ${ }^{18} \mathrm{~F}-\mathrm{DOPA}$ positron emission tomography (PET) to investigate the dopamine synthesis capacity (DSC) in the striatum [14]. In other studies, it was found that there was increased DSC in patients with SZ compared to controls [15, 16], and a direct correlation was identified between the baseline striatal DSC and the subsequent clinical response to antipsychotics [17]. An increased DSC has also been observed in patients who have a clinically determined high risk of psychosis, psychotic bipolar affective disorder (BAD) and temporal epilepsy with psychotic features $[18,19]$. In addition to increased DSC, increased occupancy of $D_{2}$ receptors by dopamine was reported in both first-episode neuroleptic-naïve patients and in previously treated chronic SZ patients experiencing an episode of illness exacerbation [20]. Elevation of stimulated dopamine release was associated with psychotic symptoms elicited in SZ induced by amphetamine in an experiment setting [21]. These studies showed that dopamine dysregulation in SZ involved processes including presynaptic dopamine synthesis and release.

These results have led to the suggestion of a transdiagnostic role for dopamine dysregulation in the pathophysiology of psychotic disorders $[22,23]$. It is hypothesized that DSC is also elevated in patients with DD and other nonaffective psychotic disorders, from which it can be inferred that dopamine dysregulation mechanistically underpins a range of psychotic disorders.

To date, there have been few studies conducted to compare pathophysiology across all psychosis disorders, including DD. A transdiagnostic study of the DSC in psychotic patients involving DD would further our understanding of the potential role of dopamine dysregulation in the pathophysiology of psychotic disorders. Therefore, this study was aimed to compare the DSCs of patients with SZ, DD, and other psychotic disorders, as well as those of healthy subjects. It was hypothesized that patients with DD, SZ-spectrum disorder (i.e., SZ and schizophreniform disorder), or other nonaffective psychoses would exhibit increased striatal DSC, relative to healthy controls.

\section{METHODS}

Participants

Participants were recruited consecutively between November 2016 and October 2017 from an intervention service in Hong Kong that targeted patients experiencing a first episode of psychosis [24]. The study inclusion criteria were as follows: (1) an age between 18 and 65 years; (2) the ability to speak Cantonese; (3) scanning examination within 1 month of antipsychotic treatment initiation; and (4) diagnosis of a first episode of DD, SZ-spectrum disorder (including SZ and schizophreniform disorder), or other psychotic disorder (including unspecified SZ spectrum and brief psychotic disorder) according to the DSM-5 criteria (6).

Patients were age and sex matched, and categorized by their having SZ and other psychotic disorders, or being healthy controls. Healthy controls were recruited through the patients' friends, voluntary organizations, and the general population, and were screened to exclude current psychiatric conditions or a family history of psychosis.

The following exclusion criteria were applied to all subjects: affective disorders with or without psychotic symptoms, substance-induced psychosis, organic psychosis, treatment with antipsychotics for more than 1 month, history of substance abuse, diagnosis of attention-deficit hyperactivity disorder, history of stimulant intake, known history of intellectual disability or special school attendance, family history of hereditary neurological disorders (e.g., Huntington's disease, Parkinson's disease (PD)), and contraindications to PET and magnetic resonance imaging (MRI) scans.

Ethical approval

Written informed consent was obtained from all of the participants. The study was approved by the Institutional Review Board at each of the six district study sites in Hong Kong. The study was conducted in accordance with Good Clinical Practices and the Declaration of Helsinki.

\section{Assessments}

Basic demographics (age, sex, years of education, body mass index (BMI), occupation, marital status, and use of alcohol/substances) were recorded at the time of study entry. The age of onset was defined as the age at which the psychotic symptoms were first manifest, and was determined using the Retrospective Assessment of the Onset of Schizophrenia (IRAOS) [25]. The duration of untreated psychosis (DUP) was also measured using the IRAOS. The diagnoses of DD, SZ, and other psychotic disorders according to the DSM- 5 were confirmed for all participants at 6 months after the onset of the first episode. These diagnoses were made by two experienced psychiatrists blinded to the results of imaging data, and were based on a best-estimate consensus that integrated all of the available information from the face-to-face interviews, medical records, and reports by informants and case workers [26]. Some of the data were collected through face-to-face interviews with the patients and their relatives. The patients' clinical symptoms were rated at baseline and during follow-up assessments using the Positive and Negative Syndrome Scale (PANSS) [27] and the Scale for Assessment of Negative Symptoms (SANS) [28]. Affective symptoms and occupational functioning were measured using the Calgary Depression Scale for Schizophrenia (CDSS) [29] and the Social and Occupational Functioning Assessment Scale (SOFAS) [30].

\footnotetext{
${ }^{18} \mathrm{~F}$-DOPA PET/MR imaging and analysis

${ }^{18}$ F-DOPA brain PET/MRI scans were performed with a Siemens Biograph PET-MR machine at the Hong Kong \& Sanatorium Hospital Department of Nuclear Medicine and Radiology. A clinical/pragmatic approach was used for the PET/MRI scanning protocol, meaning that premedication with entacapone and carbidopa was not required, and a reduced scan time was used relative to previous studies [31]. Scanning began at $30 \mathrm{~min}$ post injection of ${ }^{18} \mathrm{~F}-\mathrm{DOPA}$, and continued for a 30-min list-mode acquisition. Simultaneous 3-T MR sequences included an ultrashort echo time for attenuation correction [32]. PET images were reconstructed using the following parameters: a threedimensional ordered subset expectation maximization, 21 subsets, 5 iterations, a $256 \times 256$ matrix, a zoom factor of 2.0 and a $3.0-\mathrm{mm}$ Gaussian filter. The regions of interest (ROIs) over the putamen, caudate, occipital lobe, and cerebellum were drawn on simultaneously acquired sagittal 3D T1-weighted MR images on three
} 
orthogonal planes by an experienced radiographer who was blinded to the diagnosis [Supplementary File 1]. These ROls were then fused with the corresponding ${ }^{18} \mathrm{~F}$-DOPA PET images to generate the time-activity curve for each ROI (MIM software, version 6.6). A simplified Patlak parametric analysis was performed to approximate the influx index rate constant for a 30-60-min acquisition window $\left(K^{\text {occ;30-60 }}\right)$ of both the putamen and caudate, using the occipital lobe as the reference region. The striatum-tocerebellum ratio was calculated by the mean standardized uptake value of the ROls during the 55-60-min interval. This scanning protocol has been successfully used in previous studies to quantify brain DSC [33-35]. High-resolution T1-weighted MRI data (repetition time: $1900 \mathrm{~ms}$, echo time: $2.37 \mathrm{~ms}$, field of view: $250 \mathrm{~mm}$, flip angle: $9^{\circ}$, echo spacing: $7.2 \mathrm{~ms}$, voxel size: $1 \mathrm{~mm}^{3}$ ) were acquired simultaneously with PET data. The individual putamen and caudate volumes were extracted from T1-weighted MR images of the brain by volumetric segmentation, using FreeSurfer software (http://surfer.nmr.mgh.harvard.edu/) [36].

Statistical analysis

Statistical analyses were performed using SPSS software, version 25.0. A chi-squared test and an independent samples $t$-test were used to compare the basic demographics of patients with different diagnoses and of healthy subjects. Comparison of clinical characteristics including DUP, medication use, PANSS, SANS, CDSS, and SOFAS were also conducted between different diagnoses, in patients only. A general linear model and post-hoc pairwise tests were used to determine the differences of the $K^{\text {occ } ; 30-60}$ value, striatal volume and striatal to cerebellum ratio. Correlation analysis of $K^{\text {occ;30-60 }}$ values and clinical characteristics segregated by diagnostic groups was conducted. Nonparametric tests were applied if the normality of the data was violated. The Benjamini-Hochberg procedure with a false discovery rate ( $q$ value) of $5 \%$ was used to manage the problem of multiple testing [37].

\section{RESULTS}

\section{Demographics}

Fifty-four subjects were recruited for this study, comprising 11 patients with DD, 12 with SZ, 12 with other psychotic disorders, and 19 healthy controls. Patients with other psychotic disorders had received diagnoses of brief psychotic disorder $(n=9)$ or unspecified SZ-spectrum disorders and other psychotic disorder $(n=3)$. The demographic details of the subjects were similar between groups (Table 1). The average ages of the subjects with $\mathrm{DD}, \mathrm{SZ}$, other psychotic disorders, or healthy controls were 44.27 $( \pm 9.11), 38.58( \pm 6.12), 38.42( \pm 15.11)$, and $42.21( \pm 6.68)$ years, respectively.

The mean injected ${ }^{18} \mathrm{~F}$-DOPA activities were $325 \pm 54 \mathrm{MBq}$ in the $\mathrm{DD}, 318 \pm 44 \mathrm{MBq}$ in the $\mathrm{SZ}, 302 \pm 60 \mathrm{MBq}$ in the other psychotic disorders, and $326 \pm 49 \mathrm{MBq}$ in the control groups. There were no significant between-group differences in the mean injected doses.

\section{Clinical presentations}

The clinical presentation details of the study groups are listed in Table 2. The DUP was longest in the DD group (32.67 \pm 37.01 months), followed by the SZ group (13.75 \pm 17.74 months), and then the other psychotic disorders group $(2.50 \pm 3.26)$, but these differences were not statistically significant. Most of the subjects had begun antipsychotic treatment prior to the scans. There were no significant between-group differences in terms of current antipsychotic drug usage, positive symptoms, negative symptoms, or total PANSS and SANS scores. Similarly, functional level and mood scores, which were assessed using the SOFAS and CDSS scores respectively, did not differ between the groups.

\begin{tabular}{|lllll}
\hline Table 1. Demographics and clinical characteristics of participants. \\
\hline $\begin{array}{l}\text { Characteristics, } \\
n(\%) / \text { Delusional } \pm \\
\text { SD }\end{array}$ & $\begin{array}{l}\text { Dehizophrenia } \\
\text { disorder } \\
(N=11)\end{array}$ & $\begin{array}{l}\text { Other } \\
(N=12)\end{array}$ & $\begin{array}{l}\text { Control } \\
\text { psychotic } \\
\text { disorders } \\
(N=12)\end{array}$ & \\
\hline Age & $44.27 \pm$ & $38.58 \pm 6.12$ & $38.42 \pm 15.11$ & $42.21 \pm 6.68$ \\
Gender & 9.11 & & & \\
$\quad$ Female & $5(45.5)$ & $7(58.3)$ & $7(58.3)$ & $11(57.9)$ \\
$\quad$ Male & $6(54.5)$ & $5(41.7)$ & $5(41.7)$ & $8(42.1)$ \\
BMl & $25.08 \pm$ & $20.68 \pm 3.02$ & $24.14 \pm 3.05$ & $22.92 \pm 3.75$ \\
Marital status & 4.45 & & & \\
$\quad$ Single & $8(72.7)$ & $6(50.0)$ & $5(41.7)$ & $6(31.6)$ \\
$\quad$ Married & $1(9.1)$ & $5(41.7)$ & $6(50.0)$ & $12(63.2)$ \\
$\quad \begin{array}{l}\text { Others } \\
\text { Years of }\end{array}$ & $2(18.2)$ & $1(8.3)$ & $1(8.3)$ & $1(5.3)$ \\
education & $10.91 \pm$ & $10.38 \pm 4.51$ & $12.83 \pm 3.20$ & $12.63 \pm 3.34$ \\
Unemployment & $8(7.27)$ & $7(58.3)$ & $7(58.3)$ & $8(44.4)$ \\
Use alcohol & $9(81.8)$ & $8(66.7)$ & $8(66.7)$ & $6(31.6)$ \\
Use substance & $1(9.1)$ & $0(0.0)$ & $1(8.3)$ & $0(0.0)$ \\
\hline
\end{tabular}

No significant differences were observed in all characteristics between different groups.

$S D$ standard deviation, BMI Body Mass Index.

$K^{\text {occ;30-60 }}$ values and putamen and caudate volumes

The volumes, striatum-to-cerebellum ratios, and $K^{\text {occ;30-60 }}$ values in both the putamen and caudate of each diagnostic group are listed in Table 3. No significant differences in either the volume or the striatum-to-cerebellum ratio were observed between the groups. The $K^{\mathrm{occ} ; 30-60}$ values in the putamen $(F(1,53)=6.904, p<0.001$, $\left.\eta_{\mathrm{p}}{ }^{2}=0.297\right)$ and caudate $\left(F(1,49)=5.516, p=0.003, \eta_{\mathrm{p}}{ }^{2}=0.273\right)$ were significantly different overall (Table 3 ). Figures 1 and 2 present the post-hoc pairwise tests of $K^{\text {occ;30-60 }}$ values with Benjamini-Hochberg correction. There were statistically significant differences in the mean $K^{\text {occ;30-60 }}$ values observed in both the putamen and caudate in the SZ group (putamen: effect size (ES) = 1.33 , corrected $p=0.002$; caudate: $\mathrm{ES}=1.40$, corrected $p=0.003$ ) and in the other psychotic disorders group (putamen: $E S=1.56$, corrected $p=0.003$; caudate: $\mathrm{ES}=1.34$, corrected $p=0.0045$ ) (Figs. 1 and 2) compared with the control group. The mean $K^{\text {occ } ; 30-60}$ values in the DD group were significantly greater in the caudate $(\mathrm{ES}=0.83$, corrected $p=0.048$ ), but marginally significant in the putamen (ES-Cohen's $d$ coefficient $=1.07$, corrected $p=$ $0.05)$ relative to the control group. The $K^{\text {occ;30-60 }}$ values did not significantly differ between the DD, SZ, and other psychotic disorders groups.

Age-adjusted correlation of clinical symptoms and cumulative antipsychotic dosages with $K^{\text {occ } ; 30-60}$ values

After adjusting for age, no significant correlations were observed of the DUP, PANSS, SANS, CDSS, and SOFAS scores with the $K^{\text {occ;30-60 }}$ values in the putamen and caudate (Supplementary Table 1). There were also no significant between-group differences in the cumulative antipsychotic dosages (Supplementary Table 2), and these did not correlate significantly with the $K^{\mathrm{occ} ; 30-60}$ values in the putamen and caudate in the overall and individual groups after adjusting for age. The details of individual patients' prescriptions of antipsychotics before scanning are listed in Supplementary Table 3.

\section{DISCUSSION}

These results demonstrate that there was an increased ${ }^{18} \mathrm{~F}$-DOPA uptake in the caudate in PET scans of patients with SZ, DD, and 
Table 2. Clinical presentation among diagnostic groups.

\begin{tabular}{|c|c|c|c|}
\hline Scales, $n(\%) /$ mean \pm SD & $\begin{array}{l}\text { Delusional disorder } \\
(N=11)\end{array}$ & $\begin{array}{l}\text { Schizophrenia } \\
(N=12)\end{array}$ & $\begin{array}{l}\text { Other psychotic disorders } \\
(N=12)\end{array}$ \\
\hline $\begin{array}{l}\text { Duration of untreated psychosis (DUP) (months), mean } \pm \text { SD } \\
\text { (median, range) }\end{array}$ & $32.67 \pm 37.01(16,1-96)$ & $13.75 \pm 17.75(4,1-50)$ & $2.50 \pm 3.26(1,0-12)$ \\
\hline Medicated (antipsychotics) & $9(81.8)$ & $9(75.0)$ & $11(91.7)$ \\
\hline \multicolumn{4}{|l|}{ PANSS } \\
\hline Positive symptoms & $18.82 \pm 4.58$ & $20.50 \pm 5.00$ & $17.25 \pm 2.90$ \\
\hline Total & $60.36 \pm 12.78$ & $65.00 \pm 10.66$ & $62.17 \pm 11.57$ \\
\hline \multicolumn{4}{|l|}{ SANS } \\
\hline Affective flattening or blunting & $1.18 \pm 2.86$ & $4.25 \pm 7.46$ & $2.17 \pm 4.99$ \\
\hline Alogia & $0.82 \pm 2.71$ & $1.17 \pm 3.01$ & $0.83 \pm 2.89$ \\
\hline Avolition-apathy & $1.82 \pm 2.82$ & $3.17 \pm 3.95$ & $1.17 \pm 2.86$ \\
\hline CDSS & $7.45 \pm 6.22$ & $6.00 \pm 4.20$ & $5.25 \pm 5.77$ \\
\hline SOFAS & $50.00 \pm 10.80$ & $53.29 \pm 9.43$ & $61.50 \pm 13.57$ \\
\hline
\end{tabular}

\begin{tabular}{|lccccc|}
\hline Table 3. The volume, striatal to cerebellum ratio and $K^{\text {occ; } 30-60}$ at the putamen and caudate of different diagnostic groups. \\
\hline Mean \pm SD & $\begin{array}{l}\text { Delusional disorder } \\
(n=11)\end{array}$ & $\begin{array}{l}\text { Schizophrenia } \\
(n=12)\end{array}$ & $\begin{array}{l}\text { Other psychotic } \\
\text { disorders }(n=12)\end{array}$ & $\begin{array}{l}\text { Control } \\
(n=19)\end{array}$ & Comparison across groups \\
\hline Volume of putamen $\left(\mathrm{mm}^{3}\right)$ & $6038.9 \pm 935.2$ & $6389.9 \pm 671.7$ & $6515.5 \pm 710.1$ & $6287.7 \pm 849.1$ & $F(1,50)=0724, p=0.542, \eta_{p}{ }^{2}=0.042$ \\
Volume of caudate $\left(\mathrm{mm}^{3}\right)$ & $6469.7 \pm 834.6$ & $6348.1 \pm 816.5$ & $6655.8 \pm 826.5$ & $6305.9 \pm 619.3$ & $F(1,50)=0.581, p=0.630, \eta_{\mathrm{p}}{ }^{2}=0.034$ \\
Putamen/cerebellum & $2.40 \pm 0.24$ & $2.48 \pm 0.22$ & $2.55 \pm 0.19$ & $2.37 \pm 0.22$ & $F(1,50)=1.773, p=0.164, \eta_{\mathrm{p}}{ }^{2}=0.096$ \\
Caudate/cerebellum & $2.16 \pm 0.21$ & $2.19 \pm 0.20$ & $2.23 \pm 0.22$ & $2.16 \pm 0.23$ & $F(1,50)=0.245, p=0.865, \eta_{\mathrm{p}}{ }^{2}=0.014$ \\
$K^{\text {occ; } 30-60}$ at putamen & $0.00775 \pm 0.00085$ & $0.00832 \pm 0.00132$ & $0.00818 \pm 0.00090$ & $0.00693 \pm 0.00067$ & $F(1,53)=6.904, p<0.001, \eta_{p}{ }^{2}=0.297$ \\
$K^{\text {occ; } 30-60}$ at caudate & $0.00717 \pm 0.00107$ & $0.00762 \pm 0.00096$ & $0.00726 \pm 0.00045$ & $0.00638 \pm 0.00081$ & $F(1,49)=5.516, p=0.003, \eta_{p}{ }^{2}=0.273$ \\
\hline
\end{tabular}

other psychotic disorders, relative to healthy controls. Patients with SZ and other psychotic disorders also had significantly greater ${ }^{18} \mathrm{~F}$-DOPA uptake in the putamen, compared to healthy controls. To the best of our knowledge, this study is the first to report increased ${ }^{18} \mathrm{~F}$-DOPA uptake in patients with $\mathrm{DD}$. The results support our hypothesis, DD, SZ-spectrum disorder (i.e., SZ and schizophreniform disorder), or other nonaffective psychoses would exhibit increased striatal DSC comparing with healthy controls, and expand on previous findings from patients with SZ to include related nonaffective psychotic disorders [19, 22]. Notably, our findings support the suggestion for a transdiagnostic role of abnormal dopamine synthesis as a common pathophysiological mechanism underlying psychosis [14]. An increased DSC has also been reported in people considered to face an ultra-high risk of psychosis, and was shown to increase progressively with the development of psychosis [38]. In addition, increases in DSC were observed in patients with BAD who exhibited psychotic features. A significant association was observed between the DSC and psychotic symptoms in patients with BAD after controlling for manic symptoms [19]. Moreover, significant increases in the DSC were observed in patients with temporal lobe epilepsy and psychosis, when compared to patients without psychosis and healthy controls [36].

Our results also demonstrate increases in the DSC across groups of patients with SZ, DD, and other forms of psychosis. These observations suggest an association of dopamine synthesis with psychotic symptoms, rather than with a specific diagnosis. However, we did not detect any significant correlation between DSC and the severity of psychotic symptoms as measured by PANSS (Supplementary Table 1). This lack of significance may be attributable to the relatively small sample size in each group of patients. Future studies should aim to clarify this issue.

Besides, the level of dopamine dysregulation may not be equal within the regions of striatum. In our study, DD groups showed significantly elevated DSC in caudate but marginally significant in putamen compared with control group. In the previous casecontrol study, it showed that the level of dopaminergic hyperactivity was more pronounced in the precommissural dorsal caudate region of striatum in SZ compared with other parts of striatum [39].

To date, few studies have been performed to examine the pathophysiology of DD. Moreover, aside from clinical studies, only a small number of studies have been conducted to compare brain structures and genetic and molecular differences in DD patients. In a recent study it was found that DD patients had distinct structural and functional brain changes when compared with healthy controls [40]. In another study, structural MRI differences were found between DD and late-onset SZ, wherein patients with DD were found to have larger lateral ventricles, relative to patients with SZ and controls [41]. The observed DSC 


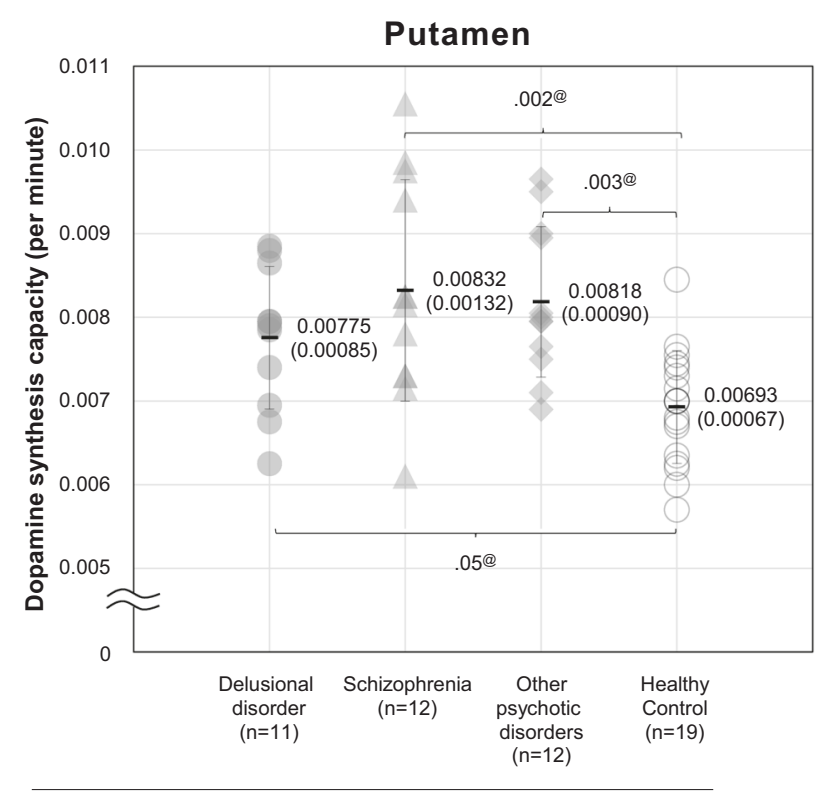

@ The $p$-value was corrected using Benjamini-Hochberg procedure.

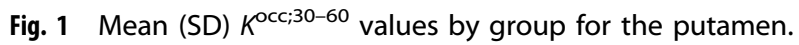

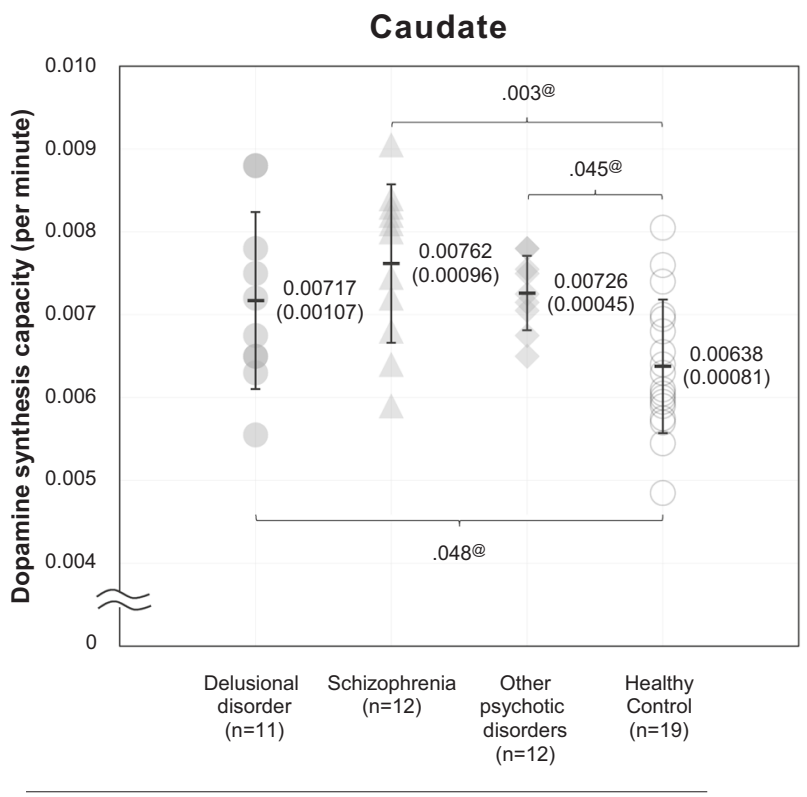

@ The $p$-value was corrected using Benjamini-Hochberg procedure.

Fig. 2 Mean (SD) $K^{\text {occ;30-60 }}$ values by group for the caudate.

dysregulation in patients with DD in our study was consistent with the findings of a previous study on dopamine metabolites and polymorphisms in the genes encoding the dopamine receptor and tyrosine hydroxylase in DD patients. Both the levels of dopamine metabolites and the genetic polymorphisms induced a hyperdopaminergic state in affected patients, particularly those with the persecutory type of DD [42]. These observations agree with our findings, as subjects with DD exhibited increased dopamine synthesis, compared to healthy controls. Our results suggest that the dopamine dysregulation observed in DD is similar to that observed in SZ and other psychotic disorders. Therefore, dopamine dysregulation may be a common pathway to psychosis [43].
Strengths and limitations

One strength of our study was the recruitment of patients who had experienced a first episode of psychosis and had no history of chronic antipsychotic therapy. This criterion reduced the potential influence of antipsychotics on the DSC.

One limitation in this study is the different methodology of the scanning protocol and premedication compared to previous ${ }^{18} \mathrm{~F}$-DOPA studies of psychosis. Most previous studies used the premedication carbidopa, a peripheral aromatic acid decarboxylase inhibitor, and entacapone, a peripheral catechol-O-methyltransferase inhibitor, administered $1 \mathrm{~h}$ before the scan to prevent the formation of peripheral ${ }^{18} \mathrm{~F}$-DOPA metabolites. These premedications were shown to increase the signal-to-noise ratio (SNR) [44], such that PET sensitivity may be reduced without premedication, possibly leading to incorrect detection of no significant differences in DSC across various types of psychosis groups. However, premedication was not used in a recent ${ }^{18} \mathrm{~F}$-DOPA study for SZ during the remission of positive symptoms, and consistent results with good reliability and comparability to studies that used premedication were obtained [45]. Thus, we believe that a reduced SNR does not alter the main result of the current study, which is that there was increased ${ }^{18} \mathrm{~F}$-DOPA uptake in the striatum of patients with SZ, DD, and other psychotic disorders, relative to healthy controls.

Furthermore, a 90-min acquisition window was used as the standard method in most of the previous ${ }^{18} \mathrm{~F}$-DOPA studies for psychosis. We note that our scanning protocol did not include the uptake period, and therefore has a much shorter acquisition time compared to the standard method. Despite the growing evidence that using shorter scanning protocols yields good reliability [45-47], this is the first psychosis research study using a $30-\mathrm{min}$ acquisition window starting $30 \mathrm{~min}$ after injection, with a reference region of the occipital lobe instead of the cerebellum. These differences in acquisition method and reference region may affect the reliability and the interpretation of these results when compared to previous studies, which is also a limitation of this study.

In contrast, the acquisition method and reference region chosen were based those used in previous ${ }^{18} \mathrm{~F}$-DOPA studies for PD $[33,34]$. Another ${ }^{18} \mathrm{~F}-\mathrm{DOPA}$ study for PD also used a shorter scanning time, in the form of the dual-time-point method. Using the occipital lobe as a reference region provides a valid approximation of the standard long-scanning protocol [48]. Both the cerebellum and occipital cortex are recommended as reference regions, because O-methyl-F-DOPA is assumed to be the sole labeled metabolite in these regions [49]. However, the abnormality of DSC in PD is well established; the existence of DSC abnormality in psychosis, and particularly in DD, has yet to be fully proven.

In a recent study it was found that DSC determined using short postinjection periods agreed closely with the data acquired from a full dynamic scan (Santangelo et al. In submission). The new short acquisition (10-15 min) scanning protocol was validated with three data sets. The first data set comprised 29 first-episode psychosis patients and 14 age-matched healthy subjects as controls. The second data set comprised 12 treatment nonresponsive and 12 treatment-responsive patients with $\mathrm{SZ}$, and 12 age-matched healthy subjects as controls. The third data set comprised eight healthy controls who were scanned twice, 2 years apart. It was concluded that a short ${ }^{18} \mathrm{~F}$-DOPA PET imaging protocol with a simplified index of F-DOPA uptake (Standardized Uptake Value Ratio), defined as the ratio of the tracer activity in the striatum to that in the cerebellum, provided a reliable and reproducible measure of dopamine synthesis, and captured a full picture of the biodistribution dynamics of ${ }^{18} \mathrm{~F}$-DOPA. Thus, this new acquisition protocol appears suitable and clinically advantageous (i.e., enabling reduced scanning time and improved patient comfort), but must be further validated using standard methods. 
Another potential limitation of the current study was the use of antipsychotics by some of the participants, although the patients began using these drugs less than 4 weeks prior to their scans. It has been found that the DSC was unaltered by a 5-week course of antipsychotic treatment [50], whilst in an earlier report a reduction in ${ }^{18} \mathrm{~F}$-DOPA uptake in response to a relatively high dose of haloperidol was observed [51]. We note that the doses of antipsychotics in this study were much lower than those in the latter study but similar to those in the former study. Further studies have reported that the DSC remains elevated in SZ patients even after chronic treatment with antipsychotics and the achievement of remission [52,53]. These observations suggest that antipsychotic treatment is unlikely to explain the observed effects in this study, and in fact may have reduced DSC differences between the patients and controls. Moreover, we found no correlation between the dose of antipsychotics and the $K^{\text {occ;30-60 }}$ value (Supplementary Table 2). Therefore, the observed between-group differences were not likely to have been caused by antipsychotic therapy.

Moreover, in the SZ and other psychotic disorders groups, but not in the DD group, we observed a significantly elevated DSC compared with controls in the putamen. This may indicate that there were differences in the extent and brain locations of dopamine dysregulation across these groups. The However, our study was not designed to detect differences between diagnostic groups, and it lacked sufficient power to detect small DSC differences between the DD and SZ groups. Further studies that aim to clarify this issue will require larger patient samples.

Smoking may also affect the DSC, as suggested in previous studies, but we collected no data on the cigarette use of participants, which may be a limitation. However, the effect of smoking on DSC is not consistent $[54,55]$. In addition, it was not found that cigarette smoking significantly the results of previous PET studies on psychosis $[45,56]$.

Our findings indicate that although differences in the clinical profiles of DD and SZ patients suggest that these are distinct diagnostic entities, both disorders are associated with dopamine dysregulation in the striatum. Notably, dopamine dysregulation may not be restricted to this area in $\mathrm{DD}$, but may also involve the prefrontal cortex, as seen in patients with SZ, which could lead to executive function deficits and cognitive biases [57]. Further PET studies of dopamine synthesis in the prefrontal cortex are needed to explore this intriguing possibility.

\section{CONCLUSION}

Our findings indicate that the dysregulated dopamine synthesis observed in SZ and other psychotic disorders is also observed in DD. This finding supports the possible transdiagnostic utility of abnormal dopamine synthesis in clinical assessments of psychosis, in that this abnormal biochemical function may be an underlying molecular mechanism of all psychoses. It follows that this could account for the similar clinical efficacy of antipsychotics for treating DD and SZ. Further studies of the DSC of patients with DD are needed to determine and verify any DSC differences between patients with this and other psychotic disorders.

\section{FUNDING AND DISCLOSURE}

This work was supported by the Food and Health Bureau of The Government of the Hong Kong Special Administrative RegionHealth and Medical Research Fund (HMRF) (Reference no. 13142781). CS has received speaker honoraria from Otsuka and DSK BioPharma. He has received funding from Otsuka and participated in paid advisory boards for Janssen and SDK BioPharma. He has received funding from Otsuka and DSK BioPharma to attend conferences. $\mathrm{ODH}$ has received investigator-initiated research funding from and/or participated in advisory/speaker meetings organized by Angellini, Astra-Zeneca, Autifony, Biogen, BMS, Eli Lilly,
Heptares, Janssen, Lundbeck, Lyden-Delta, Otsuka, Servier, Sunovion, Rand, and Roche. Neither ODH or his family have been employed by or have holdings/a financial stake in any pharmaceutical company. Other authors have declared that there are no competing interests in relation to the subject of this study. The authors declare no competing interests.

\section{ACKNOWLEDGEMENTS}

We would like to thank all of the subjects who took part in this study, staff from the Department of Psychiatry in Hospital Authority and staff from the Hong Kong Sanatorium \& Hospital. We also thank Dr. Peter Mckenna and Dr. Edith Pomarol-Clotet for their expert advice on our study.

\section{AUTHOR CONTRIBUTIONS}

CPWC, CYHE, ODH, and CWC contributed to the written recording and interpretation of data. HCL, GLG, SYN, LYLE, AYKMP, CS, BS, and MV contributed to the acquisition and analysis of data. CYHE, CKWS, LHMW, HLMC, MKFH, SPC, and ODH contributed to the conception and design of the work.

\section{ADDITIONAL INFORMATION}

Supplementary Information accompanies this paper at (https://doi.org/10.1038/ s41386-020-0740-x).

Publisher's note Springer Nature remains neutral with regard to jurisdictional claims in published maps and institutional affiliations.

\section{REFERENCES}

1. Kendler KS. The nosologic validity of paranoia (simple delusional disorder): a review. Arch Gen Psychiatry. 1980;37:699-706.

2. American Psychiatric Association. Diagnostic and statistical manual of mental disorders. 5th ed. Washington, DC: American Psychiatric Publishing; 2013.

3. Perälä J, Suvisaari J, Saarni SI, Kuoppasalmi K, Isometsä E, Pirkola S, et al. Lifetime prevalence of psychotic and bipolar I disorders in a general population. Arch Gen Psychiatry. 2007;64:19-28.

4. Chang WC, Wong CSM, Chen EYH, Lam LCW, Chan WC, Ng RMK, et al. Lifetime prevalence and correlates of schizophrenia-spectrum, affective, and other nonaffective psychotic disorders in the Chinese adult population. Schizophr Bull. 2017;43:1280-90

5. Díaz-Caneja CM, Cervilla JA, Haro JM, Arango C, de Portugal E. Cognition and functionality in delusional disorder. Eur Psychiatry. 2019;55:52-60.

6. Kendler KS. Demography of paranoid psychosis (delusional disorder). Arch Gen Psychiatry. 1982;39:890.

7. Marneros A, Pillmann F, Wustmann T. Delusional disorders-are they simply paranoid schizophrenia? Schizophr Bull. 2010;38:561-8.

8. Peralta V, Cuesta MJ. Delusional disorder and schizophrenia: a comparative study across multiple domains. Psychol Med. 2016;46:2829-39.

9. Kendler KS, Hays P. Paranoid psychosis (delusional disorder) and schizophrenia: a family history study. Arch Gen Psychiatry. 1981;38:547-51.

10. Lepping P, Russell I, Freudenmann RW. Antipsychotic treatment of primary delusional parasitosis. Br J Psychiatry. 2007;191:198-205.

11. González-Rodríguez A, Molina-Andreu O, Penadés R, Bernardo M, Catalán R. Effectiveness of long-acting injectable antipsychotics in delusional disorders with nonprominent hallucinations and without hallucinations. Int Clin Psychopharmacol. 2014;29:177-80.

12. Mews MR, Quante A. Comparative efficacy and acceptability of existing pharmacotherapies for delusional disorder. J Clin Psychopharmacol. 2013;33:512-9.

13. Ruiz V, Torra $M$, Bernardo $M$. Antipsychotic response in delusional disorder and schizophrenia: a prospective cohort study. Actas Esp Psiquiatr. 2016;44:125-35.

14. Howes O, McCutcheon R, Stone J. Glutamate and dopamine in schizophrenia: an update for the 21st century. J Psychopharmacol. 2015;29:97-115.

15. Howes OD, Kambeitz J, Kim E, Stahl D, Slifstein M, Abi-Dargham A, et al. The nature of dopamine dysfunction in schizophrenia and what this means for treatment. Arch Gen Psychiatry. 2012;69:776-86.

16. Hietala J, Syvälahti E, Vilkman H, Vuorio K, Räkköläinen V, Bergman J, et al. Depressive symptoms and presynaptic dopamine function in neuroleptic-naive schizophrenia. Schizophr Res. 1999;35:41-50.

17. Jauhar $S$, Veronese $M$, Nour MM, Rogdaki $M$, Hathway $P$, Turkheimer FE, et al. Determinants of treatment response in first-episode psychosis: an 18 F-DOPA PET study. Mol Psychiatry. 2019;24:1502-12. 
18. Fusar-Poli $P$, Howes OD, Allen $P$, Broome $M$, Valli I, Asselin MC, et al. Abnormal prefrontal activation directly related to pre-synaptic striatal dopamine dysfunction in people at clinical high risk for psychosis. Mol Psychiatry. 2009;16:67-75.

19. Reith J, Benkelfat C, Sherwin A, Yasuhara Y, Kuwabara H, Andermann F, et al. Elevated dopa decarboxylase activity in living brain of patients with psychosis. Proc Natl Acad Sci USA. 1994:91:11651-4.

20. Abi-Dargham A, Rodenhiser J, Printz D, Zea-Ponce Y, Gil R, Kegeles LS, et al. Increased baseline occupancy of D2 receptors by dopamine in schizophrenia. Proc Natl Acad Sci USA. 2000;97:8104-9.

21. Laruelle M, Abi-Dargham A, Van Dyck CH, Gil R, D'Souza CD, Erdos J, et al. Single photon emission computerized tomography imaging of amphetamine-induced dopamine release in drug-free schizophrenic subjects. Proc Natl Acad Sci USA. 1996;93:9235-40.

22. Jauhar S, Nour MM, Veronese M, Rogdaki M, Bonoldi I, Azis M, et al. A test of the transdiagnostic dopamine hypothesis of psychosis using positron emission tomographic imaging in bipolar affective disorder and schizophrenia. JAMA Psychiatry. 2017;74:1206.

23. Ashok AH, Marques TR, Jauhar S, Nour MM, Goodwin GM, Young AH, et al. The dopamine hypothesis of bipolar affective disorder: the state of the art and implications for treatment. Mol Psychiatry. 2017;22:666.

24. Hui CL, Chang WC, Chan SK, Lee EH, Tam WW, Lai DC, et al. Early intervention and evaluation for adult-onset psychosis: the JCEP study rationale and design. Early Interv Psychiatry. 2013;8:261-8.

25. Häfner H, Riecher-Rössler A, Hambrecht M, Maurer K, Meissner S, Schmidtke A, et al. IRAOS: an instrument for the assessment of onset and early course of schizophrenia. Schizophr Res. 1992;6:209-23.

26. Leckman JF. Best estimate of lifetime psychiatric diagnosis. Arch Gen Psychiatry. 1982;39:879.

27. Kay SR, Fiszbein A, Opler LA. The Positive and Negative Syndrome Scale (PANSS) for schizophrenia. Schizophr Bull. 1987;13:261-76.

28. Andreasen NC. Negative symptoms in schizophrenia: definition and reliability. Arch Gen Psychiatry. 1982;39:784-8.

29. Addington D, Addington J, Maticka-Tyndale E. Assessing depression in schizophrenia: the calgary depression scale. Br J Psychiatry. 1993;163:39-44.

30. Saraswat N, Rao K, Subbakrishna D, Gangadhar B. The Social Occupational Functioning Scale (SOFS): a brief measure of functional status in persons with schizophrenia. Schizophr Res. 2006;81:301-9.

31. Egerton A, Chaddock CA, Winton-Brown TT, Bloomfield MA, Bhattacharyya S, Allen $\mathrm{P}$, et al. Presynaptic striatal dopamine dysfunction in people at ultrahigh risk for psychosis: findings in a second cohort. Biol Psychiatry. 2013; 74:106-12.

32. Roy S, Wang WT, Carass A, Prince JL, Butman JA, Pham DL. PET attenuation correction using synthetic CT from ultrashort echo-time MR imaging. J Nucl Med. 2014;55:2071-7.

33. Chen S, Leung YL, Cheng T, Wong KN, Ho CL. Functional analysis of 18F-DOPA PET for the diagnosis of Parkinson's disease. J Nucl Med. 2012;53:1905

34. Chen S, Leung YL, Au Yeung KM, Cheung W, Wong KN, Cheng K, et al. Comparative study of ${ }^{18} \mathrm{~F}$-DOPA PET/MR and PET/CT in quantification accuracy and clinical applicability for Parkinson's disease: a static and dynamic data analysis. J Nucl Med. 2016;57:1830

35. Ho CL, Chan YK, Chen S, Leung YL, Mak KF, Chen YH. Patlak analysis of striatal dopamine synthesis capacity in earlier and later-onset psychotic illnesses with ${ }^{18}$ F-DOPA brain PET/MR imaging. J Nucl Med. 2018;59:553.

36. Fischl B. FreeSurfer. Neuroimage. 2012;62:774-81.

37. Benjamini $Y$, Hochberg $Y$. Controlling the false discovery rate: a practical and powerful approach to multiple testing. J R Stat Soc Ser B Stat Methodol. 1995:57:289-300.
38. Howes O, Bose S, Turkheimer F, Valli I, Egerton A, Stahl D, et al. Progressive increase in striatal dopamine synthesis capacity as patients develop psychosis: a PET study. Mol Psychiatry. 2011;16:885.

39. Kegeles LS, Abi-Dargham A, Frankle WG, Gil R, Cooper TB, Slifstein M, et al. Increased synaptic dopamine function in associative regions of the striatum in schizophrenia. Arch Gen Psychiatry. 2010;67:231-9.

40. Vicens V, Radua J, Salvador R, Anguera-Camos M, Canales-Rodríguez EJ, Sarro S, et al. Structural and functional brain changes in delusional disorder. Br J Psychiatry. 2016;208:153-9.

41. Howard RJ, Almeida O, Levy R, Graves P, Graves M. Quantitative magnetic resonance imaging volumetry distinguishes delusional disorder from late-onset schizophrenia. Br J Psychiatry. 1994;165:474-80.

42. Morimoto K. Delusional disorder: molecular genetic evidence for dopamine psychosis. Neuropsychopharmacology.2002;26:794-801.

43. Howes OD, Kapur S. The dopamine hypothesis of schizophrenia: version III-the final common pathway. Schizophr Bull. 2009;35:549-62.

44. Cumming $P$, Leger GC, Kuwabara $H$, Gjedde A. Pharmacokinetics of plasma 6[18F] fluoro-L-3, 4-dihydroxyphenylalanine ([18F] FDOPA) in humans. J Cereb Blood Flow Metab. 1994;13:668-75.

45. Avram M, Brandl F, Cabello J, Leucht C, Scherr M, Mustafa M, et al. Reduced striatal dopamine synthesis capacity in patients with schizophrenia during remission of positive symptoms. Brain. 2019;142:1813-26.

46. Cumming $P$, Gjedde A. Compartmental analysis of dopa decarboxylation in living brain from dynamic positron emission tomograms. Synapse.1998;29:37-61.

47. Deserno L, Huys QJ, Boehme R, Buchert R, Heinze HJ, Grace AA, et al. Ventral striatal dopamine reflects behavioral and neural signatures of model-based control during sequential decision making. Proc Natl Acad Sci USA. 2015;112:1595-600.

48. Lopes Alves I, Meles SK, Willemsen AT, Dierckx RA, Marques da Silva AM, Leenders $\mathrm{KL}$, et al. Dual time point method for the quantification of irreversible tracer kinetics: a reference tissue approach applied to [18F]-FDOPA brain PET. J Cereb Blood Flow Metab. 2017;37:3124-34.

49. Kumakura Y, Cumming P. PET studies of cerebral levodopa metabolism: a review of clinical findings and modeling approaches. J Neurosci. 2009;15:635-50.

50. Jauhar S, Veronese M, Nour MM, Rogdaki M, Hathway $P$, Natesan S, et al. The Effects of antipsychotic treatment on presynaptic dopamine synthesis capacity in first-episode psychosis: a positron emission tomography study. Biol Psychiatry. 2019;85:79-87.

51. Gründer G, Vernaleken I, Müller MJ, Davids E, Heydari N, Buchholz HG, et al. Subchronic haloperidol downregulates dopamine synthesis capacity in the brain of schizophrenic patients in vivo. Neuropsychopharmacology. 2002;28:787-94.

52. Demjaha A, Murray RM, McGuire PK, Kapur S, Howes OD. Dopamine synthesis capacity in patients with treatment-resistant schizophrenia. Am J Psychiatry. 2012;169:1203-10.

53. Howes OD, Williams M, Ibrahim K, Leung G, Egerton A, McGuire PK, et al. Midbrain dopamine function in schizophrenia and depression: a post-mortem and positron emission tomographic imaging study. Brain. 2013;136:3242-51.

54. Bloomfield MA, Pepper F, Egerton A, Demjaha A, Tomasi G, Mouchlianitis E, et al. Dopamine function in cigarette smokers: an [18 F]-DOPA PET study. Neuropsychopharmacology. 2014;39:2397-404.

55. Rademacher L, Prinz S, Winz O, Henkel K, Dietrich CA, Schmaljohann J, et al. Effects of smoking cessation on presynaptic dopamine function of addicted male smokers. Biol Psychiatry. 2016;80:198-206.

56. Kim E, Howes OD, Veronese M, Beck K, Seo S, Park JW, et al. Presynaptic dopamine capacity in patients with treatment-resistant schizophrenia taking clozapine: an [18F] DOPA PET study. Neuropsychopharmacology. 2017;42:941-50.

57. Ibanez-Casas I, Cervilla JA. Neuropsychological research in delusional disorder: a comprehensive review. Psychopathology. 2012;45:78-95. 